Supplement of The Cryosphere, 15, 2255-2272, 2021

https://doi.org/10.5194/tc-15-2255-2021-supplement

(C) Author(s) 2021. CC BY 4.0 License.

(c) (i)

Supplement of

\title{
Enhancement of snow albedo reduction and radiative forcing due to coated black carbon in snow
}

\section{Wei Pu et al.}

Correspondence to: Xin Wang (wxin@1zu.edu.cn)

The copyright of individual parts of the supplement might differ from the article licence. 


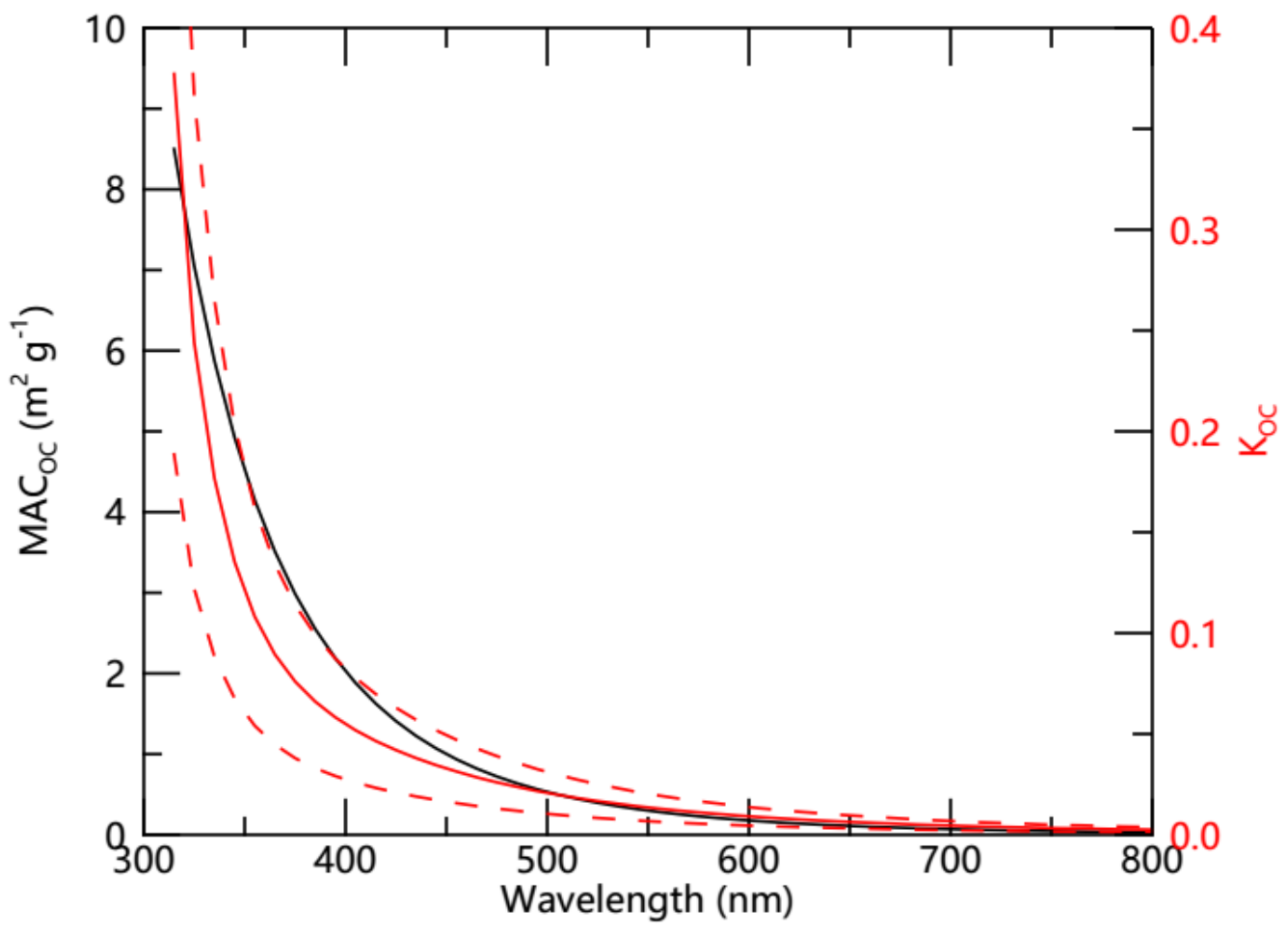

Figure S1. Mie theory calculated mass absorption coefficient (MAC) (solid black line) and imaginary refractive index (RI) (solid red line) of OC, assuming an MAC of $0.3 \mathrm{~m}^{2} \mathrm{~g}^{-1}$ at $550 \mathrm{~nm}$, a real RI of 1.55, an absorption angstrom exponent (AAE) of -6, and a particle diameter of $200 \mathrm{~nm}$ for OC. The dashed red lines represent a 50\% increase and a 50\% decrease in imaginary RI. 

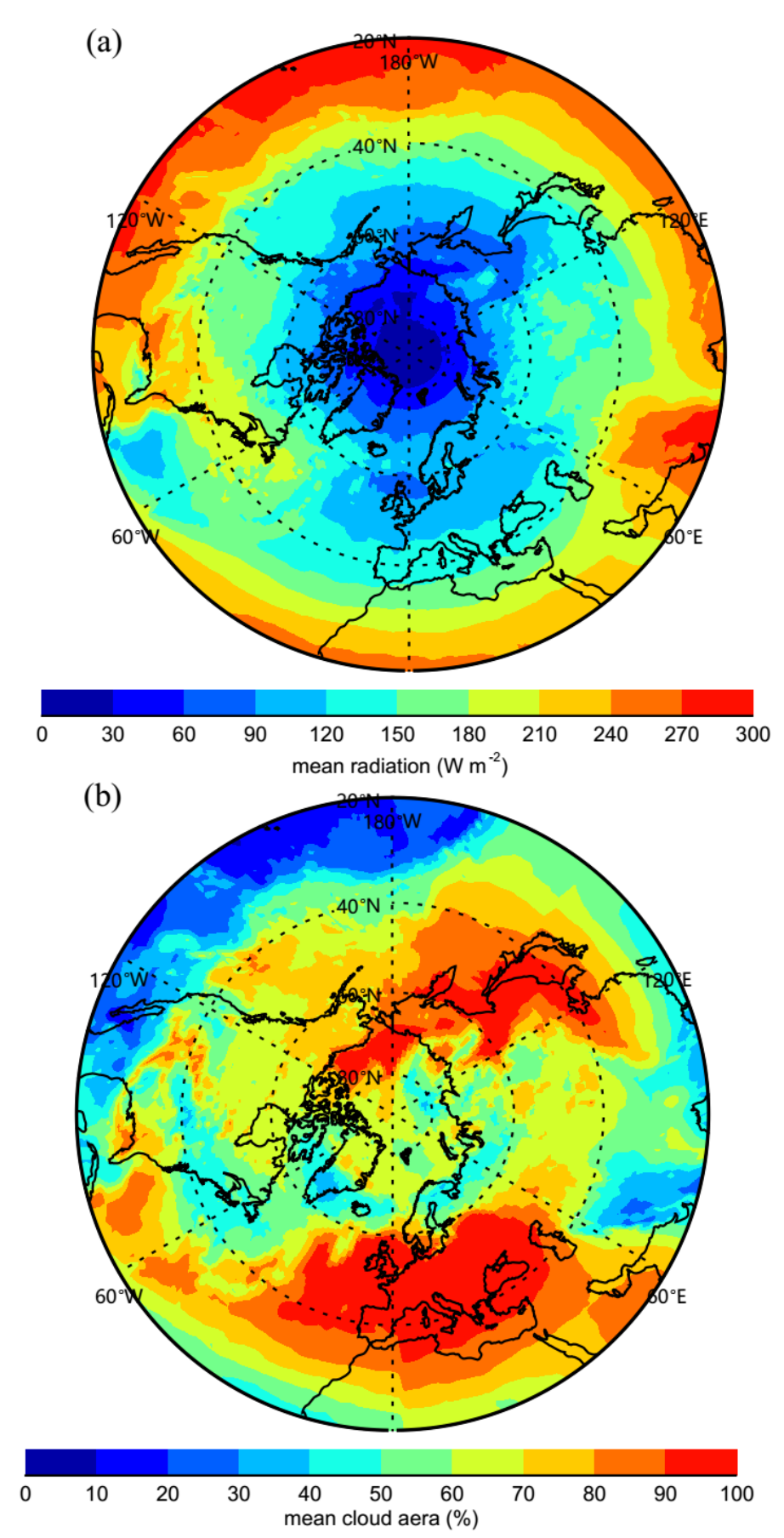

Figure S2. The 2003-2018 spatial distributions of (a) average solar flux, and (b) cloud fraction, both of which are obtained from the Clouds and the Earth's Radiant Energy System (CERES) (https://ceres.larc.nasa.gov/products.php?product=SYN1deg). 
(a) external mixing

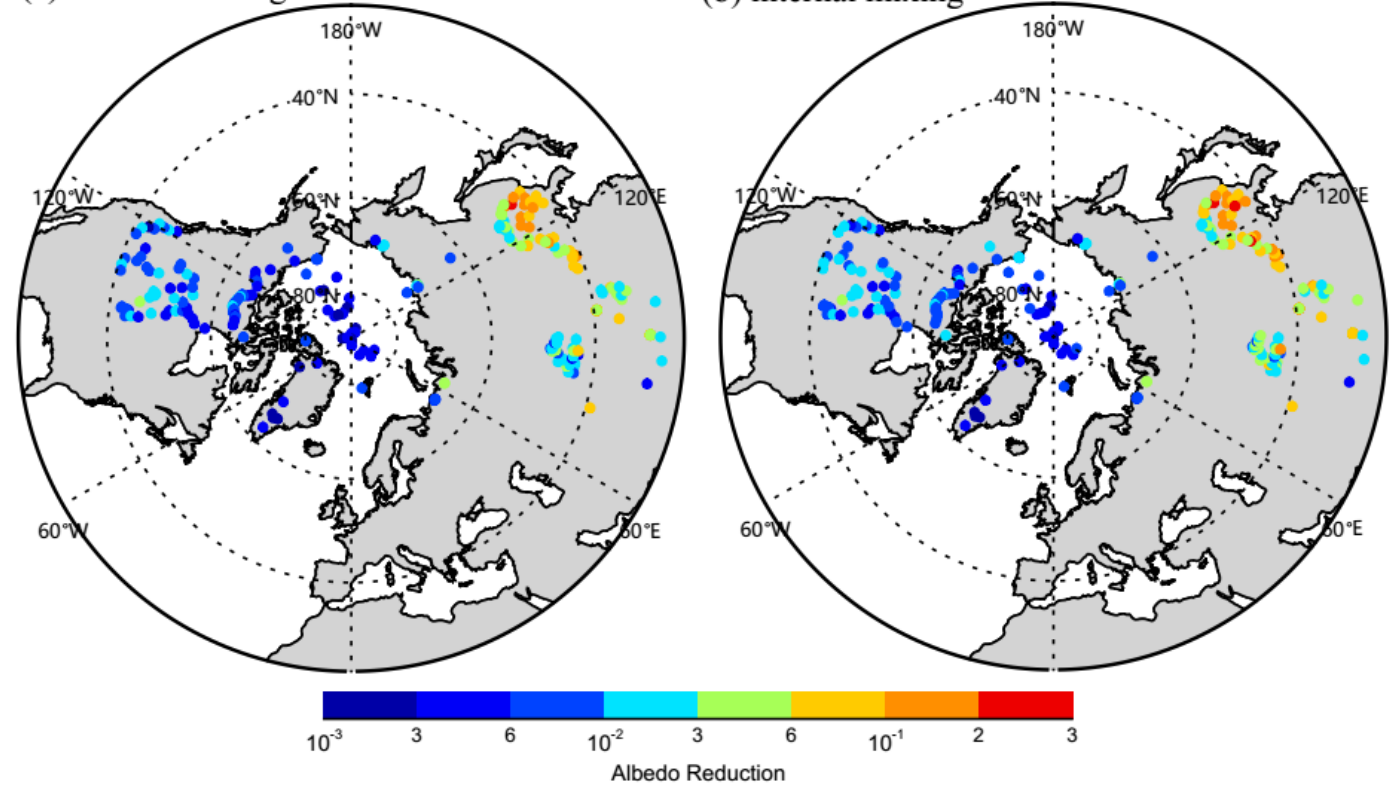

(c) external mixing

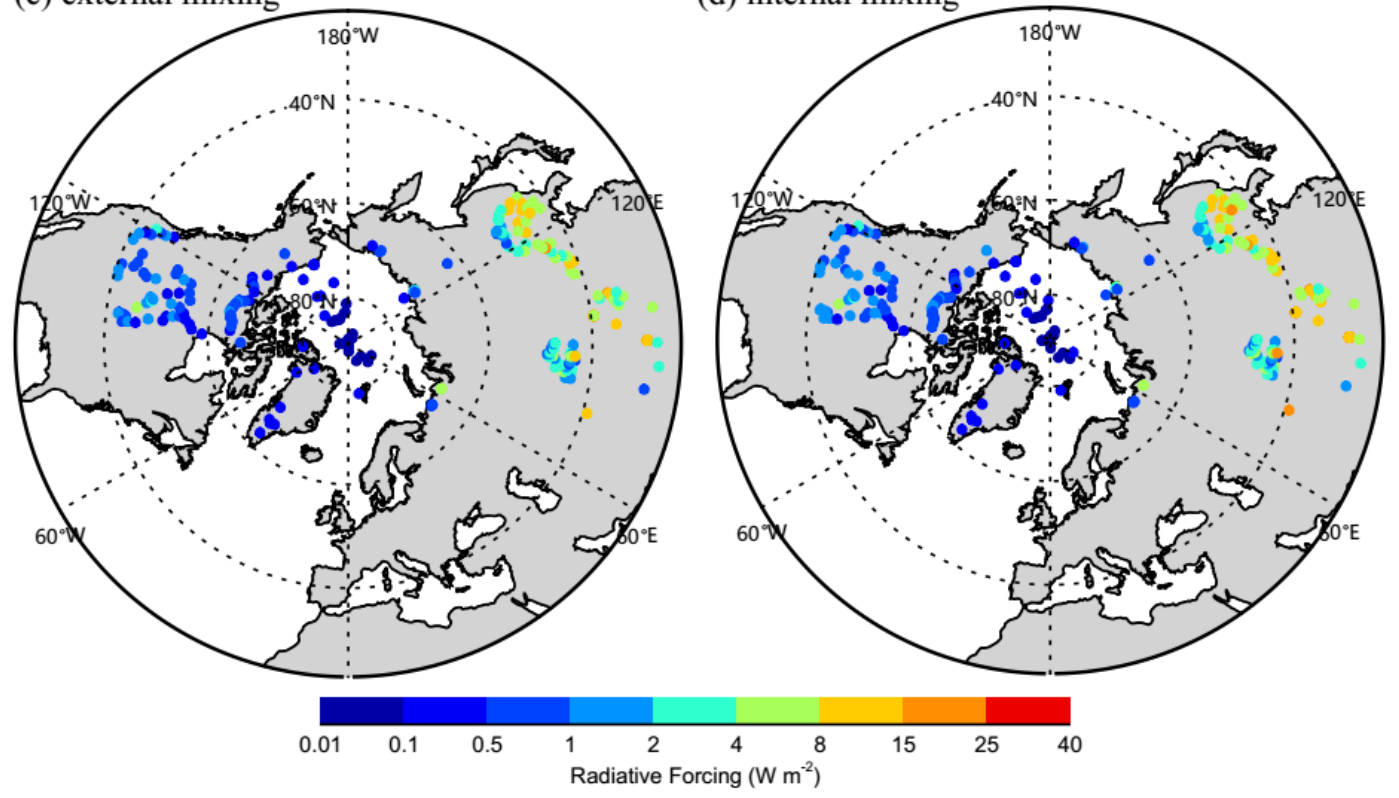

Figure S3. The spatial distributions of calculated snow albedo reductions from (a) an external mixed particle, and (b) an internal mixed particle, based on in situ measurements of fresh snow. (c) and (d) Same as (a) and (b), but for radiative forcing. 
(a) external mixing

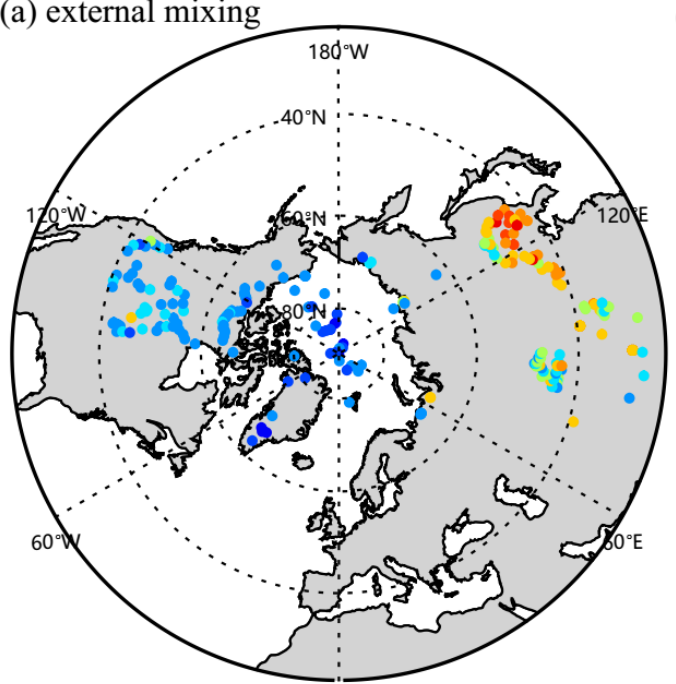

(b) internal mixing

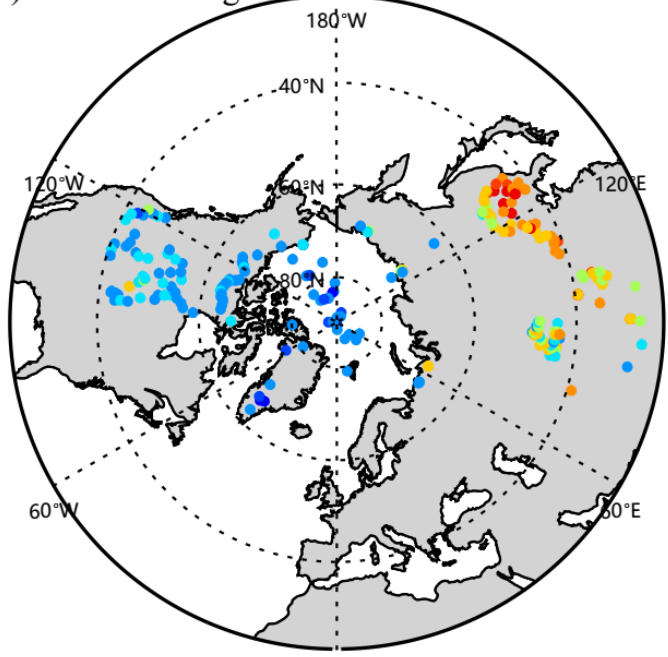

(c) external mixing

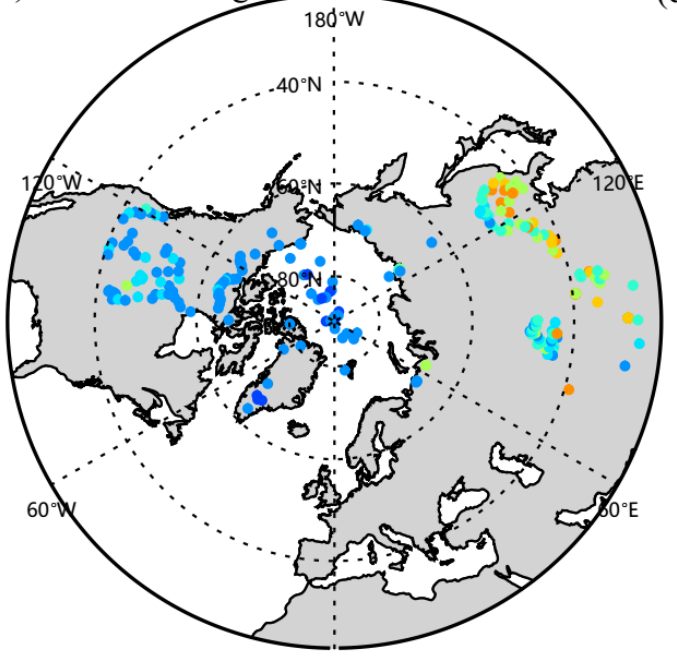

(d) internal mixing

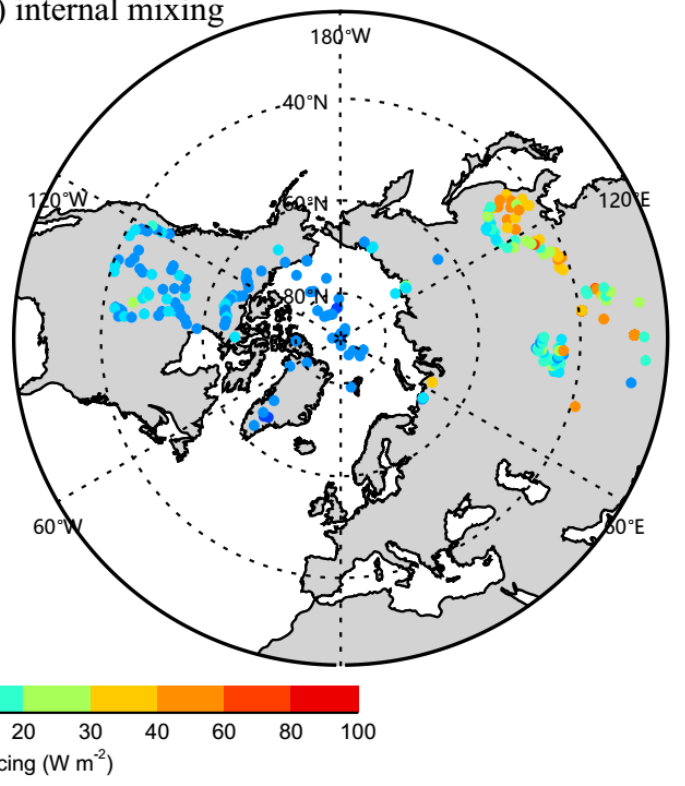

Figure S4. Same as Figure S3, but for old snow. 
Table S1. Parameterization coefficients for the ratio of broadband snow albedo of an internal mixed particle versus an external mixed particle ( $\mathrm{E}_{\alpha \text {, integrated, para }}$ ), for non-absorbing particles.

\begin{tabular}{|c|c|c|c|c|c|}
\hline $\mathrm{BC}\left(\mathrm{ng} \mathrm{g}^{-1}\right)$ & Core/shell & $a_{0}$ & $\mathrm{~b}_{0}$ & $\mathrm{~b}_{1}$ & $a_{2}$ \\
\hline \multirow{20}{*}{$0-200$} & 1.1 & $-1.4999 \mathrm{E}-04$ & $6.3289 \mathrm{E}-01$ & 4.4739E-01 & $9.9952 \mathrm{E}-01$ \\
\hline & 1.2 & $-2.8857 \mathrm{E}-04$ & $6.2817 \mathrm{E}-01$ & 4.4969E-01 & $9.9909 \mathrm{E}-01$ \\
\hline & 1.3 & $-4.1933 \mathrm{E}-04$ & $6.2416 \mathrm{E}-01$ & $4.5152 \mathrm{E}-01$ & $9.9871 \mathrm{E}-01$ \\
\hline & 1.4 & $-5.4464 \mathrm{E}-04$ & 6.2072E-01 & $4.5296 \mathrm{E}-01$ & $9.9836 \mathrm{E}-01$ \\
\hline & 1.5 & $-6.6606 \mathrm{E}-04$ & $6.1772 \mathrm{E}-01$ & 4.5407E-01 & $9.9802 \mathrm{E}-01$ \\
\hline & 1.6 & $-7.8452 \mathrm{E}-04$ & $6.1507 \mathrm{E}-01$ & $4.5490 \mathrm{E}-01$ & $9.9771 \mathrm{E}-01$ \\
\hline & 1.7 & $-9.0040 \mathrm{E}-04$ & $6.1270 \mathrm{E}-01$ & $4.5548 \mathrm{E}-01$ & $9.9740 \mathrm{E}-01$ \\
\hline & 1.8 & $-1.0134 \mathrm{E}-03$ & $6.1059 \mathrm{E}-01$ & $4.5584 \mathrm{E}-01$ & $9.9711 \mathrm{E}-01$ \\
\hline & 1.9 & $-1.1224 \mathrm{E}-03$ & $6.0875 \mathrm{E}-01$ & $4.5596 \mathrm{E}-01$ & $9.9683 \mathrm{E}-01$ \\
\hline & 2.0 & $-1.3234 \mathrm{E}-03$ & $6.0588 \mathrm{E}-01$ & 4.5542E-01 & $9.9633 \mathrm{E}-01$ \\
\hline & 2.1 & $-1.3234 \mathrm{E}-03$ & $6.0588 \mathrm{E}-01$ & 4.5542E-01 & $9.9633 \mathrm{E}-01$ \\
\hline & 2.2 & $-1.4131 \mathrm{E}-03$ & $6.0486 \mathrm{E}-01$ & $4.5475 \mathrm{E}-01$ & $9.9610 \mathrm{E}-01$ \\
\hline & 2.3 & $-1.4953 \mathrm{E}-03$ & $6.0404 \mathrm{E}-01$ & $4.5386 \mathrm{E}-01$ & $9.9589 \mathrm{E}-01$ \\
\hline & 2.4 & $-1.5704 \mathrm{E}-03$ & $6.0334 \mathrm{E}-01$ & $4.5278 \mathrm{E}-01$ & $9.9570 \mathrm{E}-01$ \\
\hline & 2.5 & $-1.6389 \mathrm{E}-03$ & $6.0269 \mathrm{E}-01$ & $4.5155 \mathrm{E}-01$ & $9.9553 \mathrm{E}-01$ \\
\hline & 2.6 & $-1.7016 \mathrm{E}-03$ & $6.0204 \mathrm{E}-01$ & 4.5019E-01 & $9.9538 \mathrm{E}-01$ \\
\hline & 2.7 & $-1.7590 \mathrm{E}-03$ & $6.0135 \mathrm{E}-01$ & 4.4873E-01 & $9.9524 \mathrm{E}-01$ \\
\hline & 2.8 & $-1.8117 \mathrm{E}-03$ & $6.0059 \mathrm{E}-01$ & $4.4718 \mathrm{E}-01$ & $9.9512 \mathrm{E}-01$ \\
\hline & 2.9 & $-1.8607 \mathrm{E}-03$ & $5.9973 \mathrm{E}-01$ & $4.4558 \mathrm{E}-01$ & $9.9502 \mathrm{E}-01$ \\
\hline & 3.0 & $-1.9072 \mathrm{E}-03$ & $5.9872 \mathrm{E}-01$ & 4.4397E-01 & 9.9493E-01 \\
\hline \multirow{20}{*}{$200-1000$} & 1.1 & $-3.6229 \mathrm{E}-05$ & 7.7791E-01 & $3.6928 \mathrm{E}-01$ & $9.9674 \mathrm{E}-01$ \\
\hline & 1.2 & $-7.0295 \mathrm{E}-05$ & $7.7151 \mathrm{E}-01$ & $3.7105 \mathrm{E}-01$ & $9.9390 \mathrm{E}-01$ \\
\hline & 1.3 & $-1.0333 \mathrm{E}-04$ & $7.6540 \mathrm{E}-01$ & $3.7252 \mathrm{E}-01$ & 9.9133E-01 \\
\hline & 1.4 & $-1.3537 \mathrm{E}-04$ & $7.6020 \mathrm{E}-01$ & $3.7370 \mathrm{E}-01$ & $9.8895 \mathrm{E}-01$ \\
\hline & 1.5 & $-1.6701 \mathrm{E}-04$ & $7.5540 \mathrm{E}-01$ & $3.7464 \mathrm{E}-01$ & $9.8670 \mathrm{E}-01$ \\
\hline & 1.6 & $-1.9838 \mathrm{E}-04$ & $7.5095 \mathrm{E}-01$ & $3.7535 \mathrm{E}-01$ & $9.8455 \mathrm{E}-01$ \\
\hline & 1.7 & $-2.2976 \mathrm{E}-04$ & $7.4668 \mathrm{E}-01$ & $3.7587 \mathrm{E}-01$ & $9.8249 \mathrm{E}-01$ \\
\hline & 1.8 & $-2.6099 \mathrm{E}-04$ & $7.4261 \mathrm{E}-01$ & $3.7619 \mathrm{E}-01$ & $9.8050 \mathrm{E}-01$ \\
\hline & 1.9 & $-2.9188 \mathrm{E}-04$ & $7.3871 \mathrm{E}-01$ & $3.7631 \mathrm{E}-01$ & $9.7860 \mathrm{E}-01$ \\
\hline & 2.0 & $-3.5250 \mathrm{E}-04$ & 7.3097E-01 & $3.7592 \mathrm{E}-01$ & $9.7512 \mathrm{E}-01$ \\
\hline & 2.1 & $-3.5250 \mathrm{E}-04$ & 7.3097E-01 & $3.7592 \mathrm{E}-01$ & $9.7512 \mathrm{E}-01$ \\
\hline & 2.2 & $-3.8097 \mathrm{E}-04$ & $7.2738 \mathrm{E}-01$ & $3.7543 \mathrm{E}-01$ & $9.7355 \mathrm{E}-01$ \\
\hline & 2.3 & $-4.0841 \mathrm{E}-04$ & $7.2379 \mathrm{E}-01$ & $3.7475 \mathrm{E}-01$ & $9.7210 \mathrm{E}-01$ \\
\hline & 2.4 & $-4.3490 \mathrm{E}-04$ & $7.2013 \mathrm{E}-01$ & $3.7391 \mathrm{E}-01$ & $9.7077 \mathrm{E}-01$ \\
\hline & 2.5 & $-4.6065 \mathrm{E}-04$ & $7.1630 \mathrm{E}-01$ & $3.7293 \mathrm{E}-01$ & $9.6955 \mathrm{E}-01$ \\
\hline & 2.6 & $-4.8583 \mathrm{E}-04$ & $7.1226 \mathrm{E}-01$ & $3.7182 \mathrm{E}-01$ & $9.6844 \mathrm{E}-01$ \\
\hline & 2.7 & $-5.1060 \mathrm{E}-04$ & $7.0796 \mathrm{E}-01$ & $3.7058 \mathrm{E}-01$ & $9.6743 \mathrm{E}-01$ \\
\hline & 2.8 & $-5.3439 \mathrm{E}-04$ & $7.0355 \mathrm{E}-01$ & $3.6925 \mathrm{E}-01$ & $9.6651 \mathrm{E}-01$ \\
\hline & 2.9 & $-5.5837 \mathrm{E}-04$ & $6.9880 \mathrm{E}-01$ & $3.6782 \mathrm{E}-01$ & $9.6568 \mathrm{E}-01$ \\
\hline & 3.0 & $-5.8229 \mathrm{E}-04$ & $6.9383 \mathrm{E}-01$ & 3.6633E-01 & $9.6492 \mathrm{E}-01$ \\
\hline
\end{tabular}


Table S2. Same as Table S1, but for absorbing particles.

\begin{tabular}{|c|c|c|c|c|c|}
\hline $\mathrm{BC}\left(\mathrm{ng} \mathrm{g}^{-1}\right)$ & Core/shell & $\mathrm{a}_{0}$ & $\mathrm{~b}_{0}$ & $b_{1}$ & $a_{2}$ \\
\hline \multirow{20}{*}{$0-200$} & 1.1 & $-1.2238 \mathrm{E}-04$ & $6.4835 \mathrm{E}-01$ & $4.3599 \mathrm{E}-01$ & $9.9957 \mathrm{E}-01$ \\
\hline & 1.2 & $-2.2834 \mathrm{E}-04$ & $6.4582 \mathrm{E}-01$ & $4.3668 \mathrm{E}-01$ & $9.9921 \mathrm{E}-01$ \\
\hline & 1.3 & $-3.2187 \mathrm{E}-04$ & $6.4400 \mathrm{E}-01$ & $4.3696 \mathrm{E}-01$ & $9.9890 \mathrm{E}-01$ \\
\hline & 1.4 & $-4.0591 \mathrm{E}-04$ & $6.4269 \mathrm{E}-01$ & $4.3691 \mathrm{E}-01$ & $9.9862 \mathrm{E}-01$ \\
\hline & 1.5 & $-4.8262 \mathrm{E}-04$ & $6.4173 \mathrm{E}-01$ & 4.3663E-01 & $9.9837 \mathrm{E}-01$ \\
\hline & 1.6 & $-5.5361 \mathrm{E}-04$ & $6.4101 \mathrm{E}-01$ & $4.3619 \mathrm{E}-01$ & $9.9814 \mathrm{E}-01$ \\
\hline & 1.7 & $-6.2006 \mathrm{E}-04$ & $6.4042 \mathrm{E}-01$ & $4.3565 \mathrm{E}-01$ & $9.9793 \mathrm{E}-01$ \\
\hline & 1.8 & $-6.8275 \mathrm{E}-04$ & $6.3992 \mathrm{E}-01$ & 4.3504E-01 & $9.9772 \mathrm{E}-01$ \\
\hline & 1.9 & $-7.4215 \mathrm{E}-04$ & $6.3946 \mathrm{E}-01$ & $4.3439 \mathrm{E}-01$ & $9.9753 \mathrm{E}-01$ \\
\hline & 2.0 & $-8.5156 \mathrm{E}-04$ & $6.3865 \mathrm{E}-01$ & 4.3294E-01 & $9.9718 \mathrm{E}-01$ \\
\hline & 2.1 & $-8.5156 \mathrm{E}-04$ & $6.3865 \mathrm{E}-01$ & 4.3294E-01 & $9.9718 \mathrm{E}-01$ \\
\hline & 2.2 & $-9.0137 \mathrm{E}-04$ & $6.3832 \mathrm{E}-01$ & $4.3213 \mathrm{E}-01$ & $9.9702 \mathrm{E}-01$ \\
\hline & 2.3 & $-9.4761 \mathrm{E}-04$ & $6.3804 \mathrm{E}-01$ & $4.3122 \mathrm{E}-01$ & $9.9687 \mathrm{E}-01$ \\
\hline & 2.4 & $-9.8999 \mathrm{E}-04$ & $6.3784 \mathrm{E}-01$ & $4.3019 \mathrm{E}-01$ & $9.9674 \mathrm{E}-01$ \\
\hline & 2.5 & $-1.0282 \mathrm{E}-03$ & $6.3772 \mathrm{E}-01$ & 4.2902E-01 & $9.9661 \mathrm{E}-01$ \\
\hline & 2.6 & $-1.0619 \mathrm{E}-03$ & $6.3770 \mathrm{E}-01$ & $4.2769 \mathrm{E}-01$ & $9.9650 \mathrm{E}-01$ \\
\hline & 2.7 & $-1.0912 \mathrm{E}-03$ & $6.3779 \mathrm{E}-01$ & $4.2619 \mathrm{E}-01$ & $9.9641 \mathrm{E}-01$ \\
\hline & 2.8 & $-1.1161 \mathrm{E}-03$ & $6.3796 \mathrm{E}-01$ & 4.2453E-01 & $9.9633 \mathrm{E}-01$ \\
\hline & 2.9 & $-1.1374 \mathrm{E}-03$ & $6.3818 \mathrm{E}-01$ & $4.2274 \mathrm{E}-01$ & $9.9626 \mathrm{E}-01$ \\
\hline & 3.0 & $-1.1561 \mathrm{E}-03$ & $6.3836 \mathrm{E}-01$ & $4.2087 \mathrm{E}-01$ & $9.9620 \mathrm{E}-01$ \\
\hline \multirow{20}{*}{$200-1000$} & 1.1 & $-3.0421 \mathrm{E}-05$ & $7.8815 \mathrm{E}-01$ & $3.6300 \mathrm{E}-01$ & $9.9711 \mathrm{E}-01$ \\
\hline & 1.2 & $-5.6630 \mathrm{E}-05$ & $7.8522 \mathrm{E}-01$ & $3.6372 \mathrm{E}-01$ & $9.9467 \mathrm{E}-01$ \\
\hline & 1.3 & $-8.0749 \mathrm{E}-05$ & 7.8117E-01 & $3.6428 \mathrm{E}-01$ & $9.9256 \mathrm{E}-01$ \\
\hline & 1.4 & $-1.0290 \mathrm{E}-04$ & $7.7776 \mathrm{E}-01$ & $3.6460 \mathrm{E}-01$ & $9.9068 \mathrm{E}-01$ \\
\hline & 1.5 & $-1.2363 \mathrm{E}-04$ & $7.7470 \mathrm{E}-01$ & $3.6471 \mathrm{E}-01$ & $9.8897 \mathrm{E}-01$ \\
\hline & 1.6 & $-1.4371 \mathrm{E}-04$ & $7.7149 \mathrm{E}-01$ & 3.6463E-01 & $9.8740 \mathrm{E}-01$ \\
\hline & 1.7 & $-1.6265 \mathrm{E}-04$ & $7.6880 \mathrm{E}-01$ & $3.6442 \mathrm{E}-01$ & $9.8593 \mathrm{E}-01$ \\
\hline & 1.8 & $-1.8054 \mathrm{E}-04$ & $7.6650 \mathrm{E}-01$ & $3.6408 \mathrm{E}-01$ & $9.8453 \mathrm{E}-01$ \\
\hline & 1.9 & $-1.9828 \mathrm{E}-04$ & $7.6396 \mathrm{E}-01$ & $3.6363 \mathrm{E}-01$ & $9.8322 \mathrm{E}-01$ \\
\hline & 2.0 & $-2.3248 \mathrm{E}-04$ & $7.5879 \mathrm{E}-01$ & $3.6238 \mathrm{E}-01$ & $9.8080 \mathrm{E}-01$ \\
\hline & 2.1 & $-2.3248 \mathrm{E}-04$ & $7.5879 \mathrm{E}-01$ & $3.6238 \mathrm{E}-01$ & $9.8080 \mathrm{E}-01$ \\
\hline & 2.2 & $-2.4901 \mathrm{E}-04$ & $7.5609 \mathrm{E}-01$ & $3.6159 \mathrm{E}-01$ & $9.7969 \mathrm{E}-01$ \\
\hline & 2.3 & $-2.6518 \mathrm{E}-04$ & $7.5324 \mathrm{E}-01$ & $3.6066 \mathrm{E}-01$ & $9.7866 \mathrm{E}-01$ \\
\hline & 2.4 & $-2.8098 \mathrm{E}-04$ & $7.5021 \mathrm{E}-01$ & $3.5960 \mathrm{E}-01$ & $9.7771 \mathrm{E}-01$ \\
\hline & 2.5 & $-2.9728 \mathrm{E}-04$ & 7.4659E-01 & $3.5837 \mathrm{E}-01$ & $9.7685 \mathrm{E}-01$ \\
\hline & 2.6 & $-3.1250 \mathrm{E}-04$ & 7.4307E-01 & $3.5700 \mathrm{E}-01$ & $9.7606 \mathrm{E}-01$ \\
\hline & 2.7 & $-3.2742 \mathrm{E}-04$ & $7.3928 \mathrm{E}-01$ & $3.5546 \mathrm{E}-01$ & $9.7536 \mathrm{E}-01$ \\
\hline & 2.8 & $-3.4211 \mathrm{E}-04$ & $7.3520 \mathrm{E}-01$ & $3.5376 \mathrm{E}-01$ & $9.7473 \mathrm{E}-01$ \\
\hline & 2.9 & $-3.5605 \mathrm{E}-04$ & $7.3105 \mathrm{E}-01$ & $3.5193 \mathrm{E}-01$ & $9.7417 \mathrm{E}-01$ \\
\hline & 3.0 & $-3.7076 \mathrm{E}-04$ & $7.2632 \mathrm{E}-01$ & $3.4998 \mathrm{E}-01$ & $9.7367 \mathrm{E}-01$ \\
\hline
\end{tabular}

